\title{
Linguatronic \\ Product-Level Speech System for Mercedes-Benz Cars
}

\author{
Paul Heisterkamp \\ DaimlerChrysler AG \\ Research and Technology \\ Speech Understanding \\ Wilhelm-Runge-Str. 11, D-89081 Ulm, Germany \\ Tel. +497315052152 \\ Paul.Heisterkamp@DaimlerChrysler.com
}

\section{INTRODUCTION}

A recent press release (Murray 2000) indicates that many car manufacturers have announced speech recognition and voiceoperated Command\&Control systems for their cars, but so far have not introduced any. They are still struggling with technology, both in reliability and pricing. The article finishes by a quote from an industry person saying:

"The reality is that today's systems are still failing in a lot of different modes. [...] But the technology will get better before it reaches the market. Right now, we just don't know when that will be."

In the light of this statement, we consider it appropriate in the context of a scientific conference, to draw the attention of the speech and language community to the fact that in-car speech is on the market, and successfully so, since fall 1996 in Germany and since May 1999 in the USA.

\section{LINGUATRONIC}

In the S-Class car of 1996, Mercedes-Benz introduced the first generation of Linguatronic. Linguatronic is the brand name used in Europe of a speech dialogue system that allows completely hands-free operation of the car's mobile phone, including number dialing (with connected digit dialog), number storing, userdefined telephone directory entry name, name dialing, and directory editing. Linguatronic I has a vocabulary of about 30 speaker-independent words (digits and control words). The second version has a vocabulary of about 300 words, and, in addition, allows for operation of comfort electronics (radio, CDplayer/changer, air condition etc). The system is now available for German, US English, UK English, Italian, Spanish, French and Swiss-German. Japanese and Dutch are currently under development.

\section{ORGANIZATION}

The basic algorithms incorporated in Linguatronic are developed by the Speech Understanding group of DaimlerChrysler Research and Technology in Ulm, Germany. These algorithms then are taken up by the Speech Processing Division of DaimlerChrysler's TEMIC business unit and put into products. These products are first marketed exclusively to the Mercedes-Benz premium brand of DaimlerChrysler, but in time, they are available to other brands and manufacturers as well. This reflects the Mercedes-Benz philosophy that safety-enhancing technology should be deployed by everybody. Thus, the speech technology from Temic is currently also available in BMW's and Audi's, with other car makers and suppliers to follow shortly with their top products..

\section{TECHNOLOGY}

The recognizer used in Linguatronic is speaker independent HMM-based. For the user definable telephone directory, an additional DTW recognizer is used. A key issue for speech technology in the car, noise reduction, is achieved by an integrated signal pre-processing that reduces the influence of noise and other sources of interference to improve the quality of the recognition. Furthermore, the system automatically and imperceptibly adapts itself to the characteristics of the speaker's voice or the acoustic background within vehicles. So, the key technology features are:

- Noise Robustness

- Echo Cancellation for hands-free telephone applications

- Continuous speaker independent speech recognition

- Speaker dependent recognition

- Active vocabulary of about 300 words

- Background adaptation

- Dynamically loadable vocabulary

Now, from the recognizer side, that doesn't seem too much different to what other speech companies and research groups offer. Yet, recognition accuracy is at a very high level. What is it, then, that makes the difference? Obviously, training of speech data plays a major role here, but others (e.g. the SpeechDat-Car project) have done major data gathering exercises as well. So, we'll point here to some items that are not naturally in the focus of the scientific community, viz. integration, co-operation and engineering. 


\section{INTEGRATION}

All upper-level cars of the major European makers these days are equipped with a data bus system, sometimes still over copper wire, but mostly using fiber optics. The Temic system supports the most common automotive bus systems like D2B, CAN, I-Bus and in the near future MOST. This means that a multitude of devices

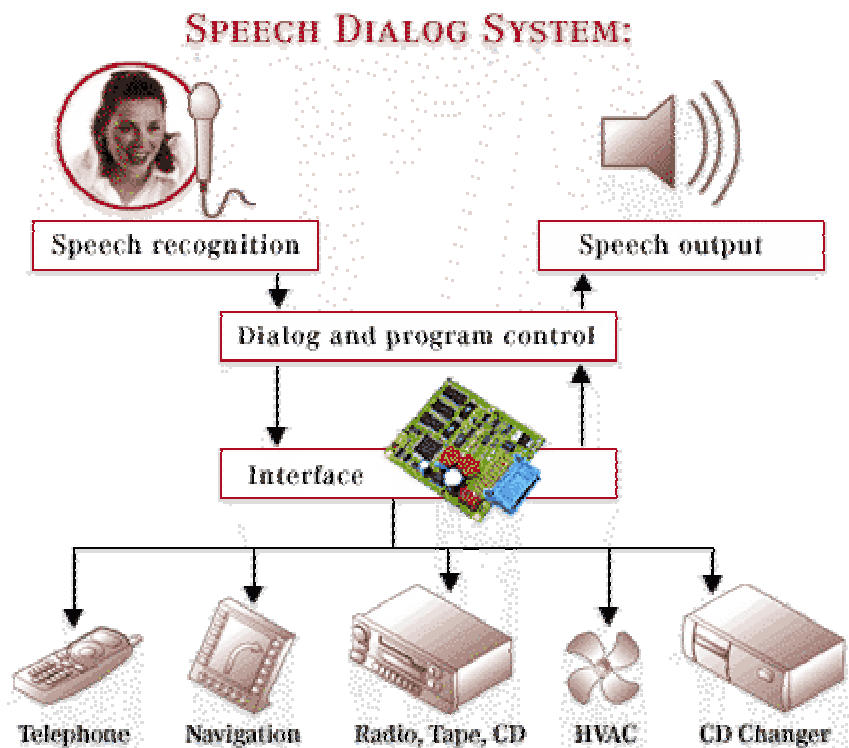
on the bus can be addressed and operated using a single and uniform speech dialogue module, and there is no need to put extra wiring in the car. Furthermore, the push-to-activate (PTA) button, muting of the audio and use of the car's loudspeakers are facilitated, and, very important, the same microphone can be used for both speech commands and telephone. The quality of the microphone (or an array), its characteristics and its positioning are extremely important.

\section{CO-OPERATION}

This kind of close integration can only be achieved in an equally close co-operation with the car manufacturer. The speech

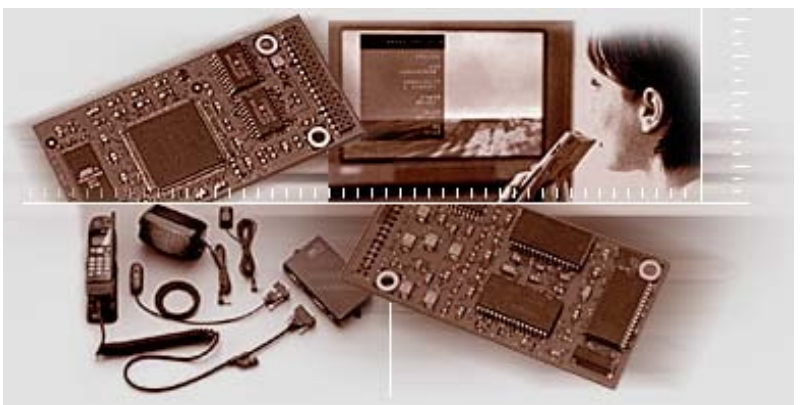

Command\&Control has to fit smoothly into the overall humanmachine-interface for the driver. From the position of the PTA button or lever, via the cancellation function to the analogous behavior of speech and tactile operation of devices, everything has to be done to ensure that speech is an integrated and natural part of the interaction. Fallback modes must be made available in case speech fails. The auto maker must also be aware that even if recognition were one-hundred-percent accurate, people do not always say what they mean or know what they can say, so there will be failures.

\section{ENGINEERING}

And, of course, the car manufacturer also knows which price people are willing to pay. The key issue now is the engineering task to deliver a product with a certain quality standard for a certain price. Besides software solutions, Temic currently offers two harware versions of its Command\&Control system for cars, one as a plug-in board the size of a credit card, the other as a selfcontained box that interfaces directly to the bus and does not require any host processor. Both not only do the recognition, but also store the speech output for the dialogue. And: at a recognition rate that convinced not only Mercedes-Benz, but also others who have a reputation for quality, Linguatronic, under the label 'voice recognition' sells in the US as part of a mobile phone package for a retail price of $\$ 480.00$; the majority of integrated phones in Mercedes-Benz cars in the US is ordered with this option. Up to the end of the year 2000, Temic has sold more than 170,000 units for automotive applications..

\section{OUTLOOK}

The scientific community is well aware that speech in cars is the enabling technology to interactively and selectively bring news and information to mobile environments without causing a safety hazard (cf., e.g. ElBoghdady 2000). Yet, we all have seen tides of enthusiasm and reluctance towards the real-life viability of speech technology. With telephony applications now firmly established, any discussion as to whether speech technology is a commercially viable option also for use in vehicles can be abbreviated by pointing at the Linguatronic example. Speech technology is there, and it will grow. More auto makers, but also system-providers like Bosch will soon be out with more complex applications, navigation address entry being the point in question, more cars

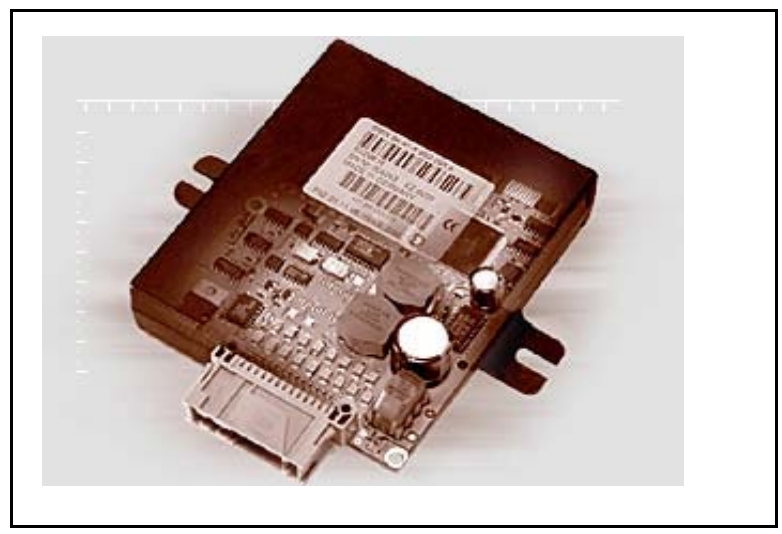

will have speech control as an option, and, in our area of research, we are pushing the limits of what can be done in research projects both internal and co-operative, e.g. in the DARPA Communicator.

\section{REFERENCES}

[1] ElBoghdady, Dina (2000): Feds fear high-tech car gear. Detnews.com, 23 January 2000.

[2] Images thanks to http://www.temic.com/speech

[3] Murray, Charles J. (2000): Automakers struggle with speech recognition technology (03 December 2000). http://www.eet.com/story/industry/systems_and_softwa re_news/OEG20001201S0109 